\title{
RIGIDITY OF INFINITE (CIRCLE) PACKINGS
}

\author{
ODED SCHRAMM
}

\section{INTRODUCTION}

The nerve of a packing is a graph that encodes its combinatorics. The vertices of the nerve correspond to the packed sets, and an edge occurs between two vertices in the nerve precisely when the corresponding sets of the packing intersect.

The nerve of a circle packing and other well-behaved packings, on the sphere or in the plane, is a planar graph. It was an observation of Thurston [Th1, Chapter 1; 13, Th2] that Andreev's theorem [An1, An2] implies that given a finite planar graph, there exists a packing of (geometric) circles on the sphere whose nerve is the given graph. We refer to this fact as the circle packing theorem. The circle packing theorem also has a uniqueness part to it: if the graph is actually (the 1-skelaton of) a triangulation, then the circle packing is unique up to Möbius transformations.

Using the circle packing theorem, Thurston proposed a method for constructing approximate maps from a given bounded planar simply connected domain to the unit disk, and conjectured that this procedure approximates the corresponding Riemann mapping. Rodin and Sullivan proved this conjecture in [RS]. One of the crucial elements in their argument is the rigidity of the hexagonal packing, the infinite packing of circles where every circle touches six others and all have the same size. (When we say that a circle packing is rigid, we mean that any other circle packing on the sphere with the same nerve is Möbius equivalent to it. In the case of the hexagonal packing, this is the same as saying that any two planar circle packings with the combinatorics of the hexagonal packing are similar.) The techniques of [RS], and the rigidity of the hexagonal packing have since been used by others [CR, He2, Ro1, Ro2, Sch1] to obtain quasiconformal and conformal maps, and to study the quality of the convergence of Thurston's approximating scheme.

The existence part of the circle packing theorem is not hard to generalize to infinite, locally finite graphs, using a geometric limit. However, the uniqueness does not hold for arbitrary (locally finite) planar triangulations. In this note we

Received by the editors November 29, 1990.

1980 Mathematics Subject Classification (1985 Revision). Primary 30C35, 52A45; Secondary 05B40, 30C60, 30C85. 
prove:

1.1. Rigidity Theorem. Let $T=T(V)$ be an infinite, planar triangulation, and let $Q=\left(Q_{v}: v \in V\right)$ be a circle packing on the sphere $S^{2}$ with nerve $T$. If $S^{2}-\operatorname{carrier}(Q)$ is at most countable, then every other circle packing on the sphere with nerve $T$ is Möbius equivalent to $Q$. (The carrier of $Q$ is the union of all the circles $Q_{v}$ and their interiors, together with the curved triangular regions in the complement of the packing.)

For example, the carrier of the hexagonal packing is the whole plane, and its complement with respect to the sphere consists of a single point, the point at infinity. Therefore the rigidity of the hexagonal packing follows from Theorem 1.1.

Similar results are proved for circle packings that almost fill an open geometric disk, and for packings consisting of convex shapes. See Theorems 5.1, 5.3, 5.4 .

The original proof of the rigidity of the hexagonal packing had relied on some rather heavy machinery. Later, $\mathrm{He}[\mathrm{He} 1]$ obtained a more direct proof with some accurate estimates. Both proofs use the theory of quasiconformal maps. The techniques of [RS] and [He1] can be used to yield rigidity results for packings with bounded valence (that is, the nerve has bounded valence) that fill the plane, but it seems unlikely that they would be sufficient to prove the rigidity theorem above. Recently, Stephenson [Ste] announced a rigidity proof that uses probabilistic arguments, but the assumptions there are even more restrictive than bounded valence.

The methods used here are mostly elementary plane topology arguments, together with simple manipulations with the Möbius group. You will find no formulae or inequalities in this paper. (But admittedly, some basic ideas of the theory of quasiconformal maps do play a role, in analogy, and in motivation. Conversely, the results here give a new perspective to the concept of the conformal modulus.) Our main tool is a theorem that we call the Incompatibility Theorem (3.1). It is a theorem about packings of sets more general than circles. In fact, the theorem is purely topological - no geometry is present in its hypotheses or its conclusions. The generality of the Incompatibility Theorem might seem a little out of place to the reader, and warrants an explanation of the background and the motivation.

In [Sch1] we generalized the existence part of the circle packing theorem to packings by more general shapes, in particular to packings of convex sets specified up to homothety, and of balls of Riemannian metrics. To give an illustration, we quote:

Convex Packing Theorem. Let $G=G(V, E)$ be a planar graph, and for each vertex $v \in V$, let $P_{v}$ be some smooth planar convex body. Then there exists a packing $\left(P_{v}^{\prime}: v \in V\right)$ in the plane, with nerve $G$, and with each $P_{v}^{\prime}$ positively homothetic to $P_{v}$. 
The fact that $P_{v}^{\prime}$ is positively homothetic to $P_{v}$ means that $P_{v}^{\prime}$ is obtained from $P_{v}$ by a positive homothety; i.e., a transformation of the form $x \rightarrow a x+b$ for some $b \in \mathbb{R}^{2}, a \in \mathbb{R}, a>0$.

The incompatibility theorem was conceived in an effort to understand the extent to which these more general packings are unique. In fact, the incompatibility theorem does give a rather painless proof for uniqueness, as well as existence, for packings under very general conditions; see [Sch2].

As the question of uniqueness of infinite circle packings is now answered in quite general circumstances, one is naturally led to the problem of existence. Consider an infinite planar triangulation $T$, and assume that $T$ has just one end. (In the complement of any finite collection of vertices of $T$ precisely one connected component is infinite.) It is easy to obtain a circle packing in the plane with nerve $T$ by taking geometric limits of finite packings. But one can say more. There is such a packing whose carrier is either the plane or the open unit disk. For no such triangulation are both of these possibilities feasible and, in any case, the packing is unique up to Möbius transformations. The existence part will be proved elsewhere, and the uniqueness part follows from the results of this paper. (This proves a conjecture of Thurston [Th2].)

\section{Definitions AND Notations}

We consider the plane $\mathbb{R}^{2}=\mathbb{C}$ as being contained in the sphere $\hat{\mathbb{C}}=S^{2}$. A circle in the plane is also a circle when thought of as a subset of $S^{2}$. When we use the term 'circle', we usually mean 'the circle together with its interior'; that is, a closed round disk.

For us, a packing means an indexed collection $P=\left(P_{v}: v \in V\right)$ of compact connected sets in the sphere $S^{2}$ with the property that the interior of each set $P_{v}$ is disjoint from the other sets $P_{w}, w \neq v$.

The nerve of the packing $P=\left(P_{v}: v \in V\right)$ is a graph whose vertex set is $V$, and that is defined by the property that there is an edge between two distinct vertices, $v, w$, if and only if the corresponding sets, $P_{v}, P_{w}$, intersect. Note that there is at most a single edge between $v, w$ in the nerve, even if $P_{v}$ and $P_{w}$ intersect in more than one place. If $G$ is a graph, then we use the notation. $G(V)$ to mean that $V$ is the vertex set of $G$.

For any $A \subset S^{2}$ let $A^{c}=S^{2}-A$ denote the complement of $A$.

We deal with packings of sets that are well behaved topologically. Most of the sets we pack are disklike: a set $A \subset S^{2}$ is disklike if it is the closure of its interior, its interior is connected, and the complement of any connected component of $A^{c}$ is a topological disk. The obvious example for a disklike set is a topological disk. A more general example is a closed ball for a path metric on $S^{2}$ (a metric in which the infimum of the lengths of paths joining any two points is the distance between them).

Let $P=\left(P_{v}: v \in V\right)$ be a packing of disklike sets on the sphere, and let $G$ be its nerve. If the intersection of any three of the sets $P_{v}$ is empty, then 
the nerve of the packing is a planar graph; that is, it can be embedded in the plane. To find such an embedding one picks an interior point $p_{v}$ in every $P_{v}$ to correspond to the vertex $v$, and one chooses a simple path from $p_{v}$ to $p_{w}$ in $P_{v} \cup P_{w}$ to correspond to an edge $v \leftrightarrow w$ in $G$. With a little care one makes sure that these curves intersect only at the vertices.

If $P$ satisfies the condition that the intersection of any three sets of $P$ is empty, then $P$ is called a nondegenerate packing. Otherwise, it is called a degenerate packing.

An embedding of a connected locally finite graph in the sphere is called a triangulation if the boundary of every connected component of the complement of the graph, with respect to the sphere, either consists of three edges of the graph or does not intersect the embedded graph at all ${ }^{1}$. The latter situation can occur only if the graph is infinite. It is easy to see that a connected locally finite graph embedded in the sphere is a triangulation if and only if the set of neighbors of every vertex is the set of vertices of a simple closed path in the graph. (We assume that there are no multiple edges or loops, and that the graph has more than three vertices.) Because this condition does not depend on the particular embedding, it follows that a planar graph is a triangulation in every embedding if it is a triangulation in some embedding. Furthermore, the embedding is unique up to homeomorphisms of the sphere (using the fact that a cycle of an embedded graph separates the sphere). Thus we freely identify the graph with the embedded graph, and we say that the graph itself is a triangulation. A connected component of the complement of the embedded graph whose boundary consists of three edges is called a triangle of the graph. Generally we ignore triangulations with fewer than four vertices.

Let $G$ be a finite planar graph, and let $B$ be a simple closed path in $G$. We say that $G$ is a triangulation with boundary $B$ if $G-B$ is connected and if $G$ has an embedding $I$ in the sphere $S^{2}$ where all the connected components of $S^{2}-I(G)$ are triangles (that is, bounded by three edges of $I(G)$ ), except possibly for one component whose boundary is $I(B)$. If $B$ has precisely four vertices, then $G$ is called a triangulation of a quadrilateral. The vertices of $B$ are the boundary vertices of $G$.

A quadrilateral is a closed topological disk $D$ in $S^{2}$ with four distinguished points $p_{0}, p_{1}, p_{2}, p_{3}$ on its boundary that are oriented clockwise with respect to the interior of $D . D_{i}$ will be used to denote the arc of the boundary of this quadrilateral that extends clockwise from $p_{i-1}$ to $p_{i}$, with $p_{4}$ standing for $p_{0}$. Such a quadrilateral is denoted by $\left(D_{1}, D_{2}, D_{3}, D_{4}\right), D\left(p_{0}, p_{1}, p_{2}, p_{3}\right)$, or just $D$, depending on convenience.

Similarly, a trilateral $D=\left(D_{1}, D_{2}, D_{3}\right)$ is defined as a topological disk with three distinct distinguished clockwise oriented points on its boundary.

\footnotetext{
${ }^{1}$ In standard terminology, the graph is the 1-skelaton of a triangulation of a spherical surface without boundary. However, as explained shortly, it is justifiable to call the graph itself a triangulation, because the triangulation is reproducible from it.
} 

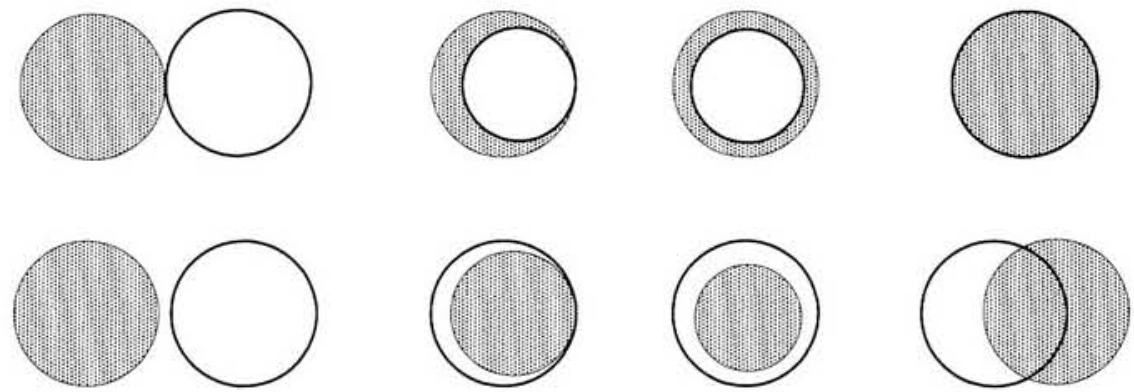

FIGURE 2.1. Topologically there are eight ways in which two circles can intersect.

Let $P$ be a nondegenerate packing in $S^{2}$ whose nerve is a triangulation $T$, possibly with boundary. When we consider the sphere as oriented, the packing $P$ naturally induces an orientation on its nerve $T$ (that is, a 'clockwise' orientation for the triangles of $T$ ). From now on, a triangulation (with boundary) means an oriented triangulation (with boundary), and when we say that a packing has a triangulation as its nerve, it is implicit that the orientation that it induces on the nerve is compatible with the orientation of the triangulation.

The motivation for the following definitions comes from the simple but very important observation that the possible patterns of intersection of two circles are very special, topologically. See Figure 2.1.

Let $D$ be a subset of the sphere $S^{2}$ and $p, q \in S^{2}$. We say that a curve $\gamma$ connects $p$ and $q$ in $D$ if the endpoints of $\gamma$ are $p, q$, and $\operatorname{relint}(\gamma) \subset D$. Here, and in the following, $\operatorname{relint}(\gamma)$ means $\gamma-\{$ its endpoints $\}$. Note that $p$ and $q$ do not have to be in $D$ to be connected by a curve in $D$; they may be in $\bar{D}-D$.

Let $A, B$ be two topological disks in the sphere. We say that $A$ cuts $B$ if there are two points in $B$-interior $(A)$ that are not connected by any curve in interior $(B-A)$. $A$ and $B$ are incompatible if $A \neq B$ and $A$ cuts $B$, or $B$ cuts $A$. Otherwise, they are compatible. See Figure 2.2 on the next page.

If $A, B$ are disklike, then they will be considered compatible if $A=B$ or $A^{\prime c}$ and $B^{\prime c}$ are unequal and compatible for every connected component $A^{\prime}$ of $A^{c}$ and every connected component $B^{\prime}$ of $B^{c}$. In other words, either $A=B$, or whenever you adjoin to $A$ all but one of the connected components of its complement and do the same to $B$, the resulting topological disks are unequal and compatible. (This definition is, in turn, compatible with the definition for the case where $A, B$ are topological disks.)

\subsection{Examples. (1) Any two circles are compatible.}

(2) More generally, homothetic strictly convex bodies in the plane are compatible.

(3) The closures of the complements of two compatible topological disks are also compatible. 

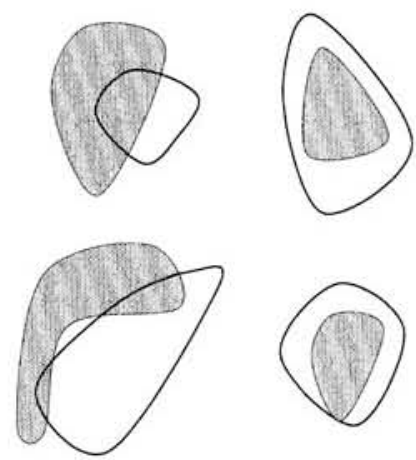

(a) Some compatible pairs.
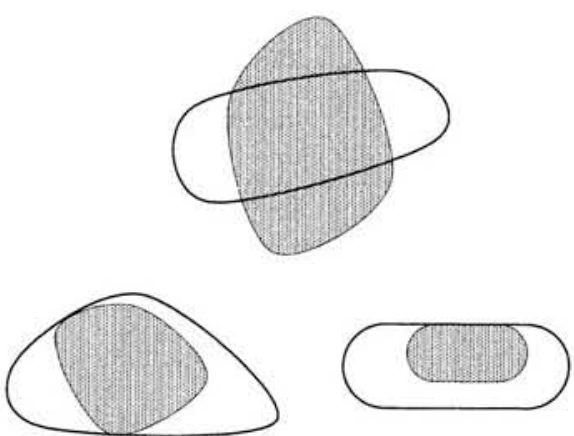

(b) Some incompatible pairs.

\section{Figure 2.2}

(4) Homothetic convex shapes that are not strictly convex can be incompatible.

(5) It is not hard to see that given a Riemannian metric on the sphere, any two metric balls of it are compatible.

And finally, some notation. Whenever $Q=\left(Q_{v}: v \in V\right)$ is a packing and $I \subset V$, the union $\bigcup_{v \in I} Q_{v}$ is abbreviated to $Q_{I}$.

\section{The INCOMPATibility Theorem}

This section is devoted mostly to the following theorem, which is our central tool.

3.1. Incompatibility Theorem. Let $T=T(V)$ be a finite triangulation of $a$ quadrilateral with boundary vertices $a, b, c, d$ in clockwise order with respect to the other vertices of $T$ (if such exist). Let $D=\left(D_{1}, D_{2}, D_{3}, D_{4}\right)$ be a quadrilateral in $S^{2}$. Suppose that $Q=\left(Q_{v}: v \in V\right)$ and $P=\left(P_{v}: v \in V\right)$ are two nondegenerate packings in $D$, both having nerve $T$. Further suppose that $Q_{v}$ and $P_{v}$ are disklike for $v \in V-\{a, b, c, d\}$; that $P_{a} \subset D_{1}, P_{b} \subset$ $D_{2}, P_{c} \subset D_{3}, Q_{d} \supset D_{4}$; and that $P_{v}$ is disjoint from $Q_{d}$ for $v \in V-\{a, c, d\}$. Then there is some vertex $v \in V-\{a, b, c, d\}$ for which $Q_{v}$ and $P_{v}$ are incompatible. See Figure 3.1.

3.2. Remarks. The quadrilateral $D$ is not an important ingredient of the situation to which the theorem applies. It is merely a frame of reference that helps us describe the relative positions of the packings $P$ and $Q$. In the situations where the theorem is applied the quadrilateral $D$ is not mentioned.

The incompatibility theorem is the packing-theoretic analogue of the concept of the conformal modulus of a quadrilateral. Lemma 5.2 is probably the corresponding analogue for the concept of the conformal modulus of an annulus.

3.3. Coalescing. Before the proof of the theorem, we describe a simple procedure that will be very useful to us. Let $T=T(V)$ be some finite planar 

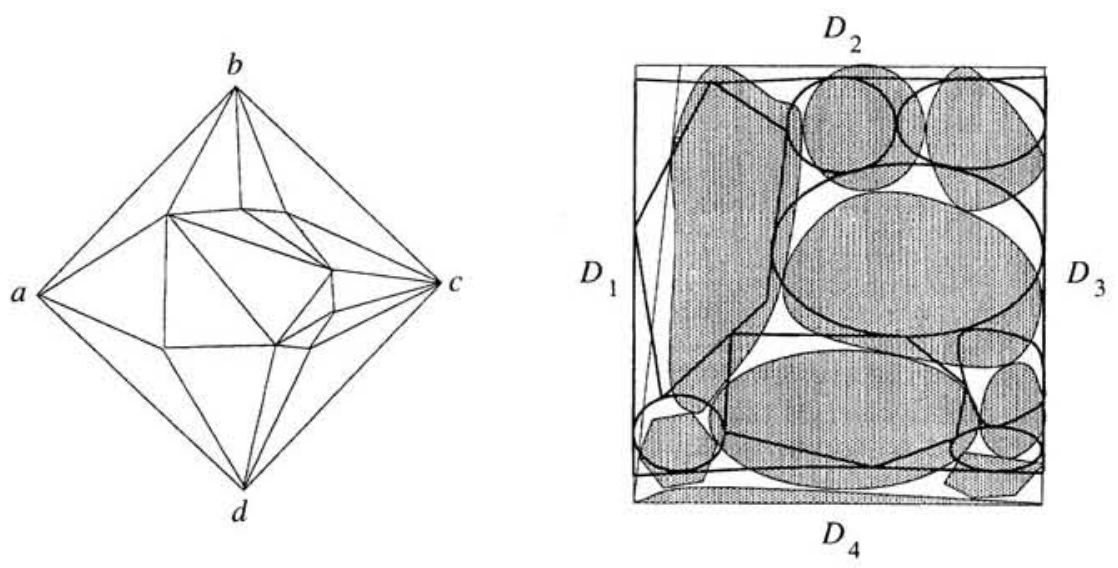

FIGURE 3.1. An incompatibility is forced. (Find it.)
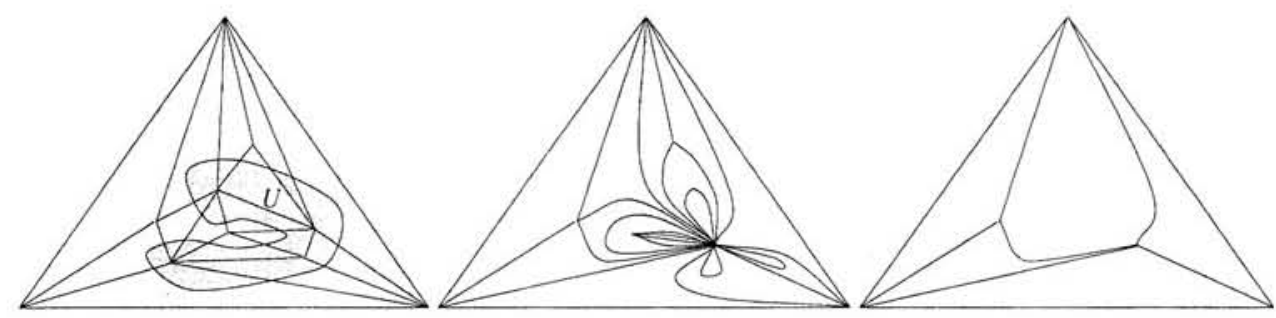

Figure 3.2. Coalescing a set of vertices. First some multiple edges are created, but these can be deleted, together with some of the vertices, to obtain a triangulation.

triangulation, and let $Q=\left(Q_{v}: v \in V\right)$ be some packing of disklike sets with nerve $T$. Suppose that $U$ is some nonempty connected subset of vertices, and suppose that $z$ is some vertex that is not in $U$. We describe what we mean by coalescing $Q_{U}$ while keeping $z$. The idea is that we want to consider the packing $\hat{Q}$ that is the same as $Q$ except for the fact that all the sets $Q_{v}, v \in U$ are united into one, $Q_{U}$. $\hat{Q}$ might not be a good packing, however, because its nerve might not be a planar triangulation, and the set $Q_{U}$ might not be disklike.

First, we look at the combinatorial picture. Coalescing the sets $Q_{v}, v \in U$ is analogous to coalescing the vertices $U$ into one. Consider a realization of $T$ in the plane. Let $\hat{T}$ be a picture obtained from $T$ by collapsing the vertices in $U$. See Figure 3.2. Generally, in $\hat{T}$ there are some loops and multiple edges, but every 2 -cell determined by $\hat{T}$ has at most three edges of $\hat{T}$ on its boundary. We want to have a triangulation without any loops or multiple edges. Therefore we must remove the excess edges from $\hat{T}$, and do this without having 2-cells with too many edges on their boundary. 
(a)

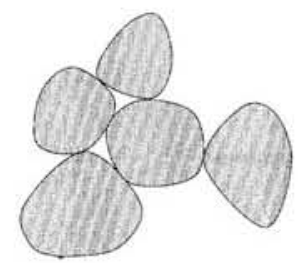

(b)

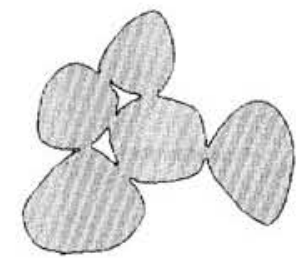

FIGURE 3.3. Initially, after coalescing, one may obtain a nondisklike set, but this is easily remedied.

Consider a loop $l$ in $\hat{T}$; that is, an edge having the same vertex at both its endpoints. If we just delete $l$ from $\hat{T}$, then we might have some resulting 2 -cell, which is a quadrilateral, and we do not want that. The loop $l$ determines two regions in $\hat{T}-l$. If we delete $l$ together with all the edges and vertices of $\hat{T}$ that are in one of these regions, then in the resulting picture all the 2-cells are still 1-, 2-, or 3-gons. Because we want to keep $z$, we delete with $l$ all the stuff that is in a region that does not contain $z$.

Similarly, we deal with multiple edges. If two edges of $\hat{T}$ have the same two vertices as endpoints, then we delete one of them, together with all the stuff in one of the two regions determined by these two edges that does not contain $z$.

It is straightforward to verify that this procedure is consistent, and that one obtains a triangulation $T^{\prime}$. (This is so provided that $z$ neighbors with some vertex that is not in $U$. If all the neighbors of $z$ are in $U$, then $T^{\prime}$ consists of two vertices and an edge between them.) In $T^{\prime}, z$ and the vertices neighboring with $z$ are still present. Let $V^{\prime}$ be the set of vertices of $T^{\prime}$, and assume that the vertex corresponding to the coalesced set $U$ is $u$.

Let $Q^{\prime}=\left(Q_{v}^{\prime}: v \in V^{\prime}\right)$ be the packing defined by $Q_{v}^{\prime}=Q_{v}, v \in V^{\prime}-\{u\}$, $Q_{u}^{\prime}=Q_{U}$. Then the packing $Q^{\prime}$ has the nerve $T^{\prime}$, and the only remaining problem is that the set $Q_{u}^{\prime}$ might not be disklike. See Figure 3.3(a). We then modify $Q_{u}^{\prime}$ slightly to make it disklike, as in Figure 3.3(b). Note that this can be done with an arbitrarily minute modification of $Q_{u}^{\prime}$, while keeping $Q^{\prime}$ as a packing with the same combinatorics.

The packing obtained in this manner is called the packing obtained from $Q$ by coalescing $U$ while keeping $z$. This procedure is done similarly for triangulations with boundary, and for infinite triangulations, provided the boundary of $U$ is finite.

Proof of Incompatibility Theorem. First we make an easy reduction to the case where all the sets $Q_{v}, P_{v}, v \in V-\{a, b, c, d\}$ are topological disks. If $Q_{v}$ $(v \neq a, b, c, d)$, say, is not a topological disk, then we can adjoin the connected components of its complement to $Q_{v}$, except for the one that intersects the other sets $Q_{w}, w \neq v$. Because $Q_{v}$ is disklike, the resulting set $Q_{v}^{\prime}$ would be a topological disk. We apply this procedure to all the $Q_{v}, P_{v}, v \in V-$ $\{a, b, c, d\}$ that are not topological disks, and obtain two packings $Q^{\prime}, P^{\prime}$ 
that satisfy the hypotheses of the theorem. If $Q_{w}^{\prime}$ and $P_{w}^{\prime} \quad(w \neq a, b, c, d)$ are incompatible, then surely, the same holds for $Q_{w}$ and $P_{w}$. We may, and do, therefore assume that the sets $Q_{v}, P_{v}, v \in V-\{a, b, c, d\}$ are topological disks.

The proof proceeds by induction on the number of vertices in $T$. The base of the induction is the case in which there are no vertices in $T$ other than $a, b, c, d$. Because $D_{1}$ and $D_{3}$ are disjoint, and $P_{a} \subset D_{1}, P_{c} \subset D_{3}$, there cannot be any edge between $a$ and $c$ in $T$. If there is an edge between $b$ and $d$, then $Q_{d}$ must intersect $Q_{b} \subset D_{2}$. This would force $Q_{d} \cap P_{b} \neq \varnothing$, because $Q_{d}$ is connected and intersects $D_{2}$ and $D_{4}$, and $P_{b}$ is connected and intersects $D_{1}$ and $D_{3}$. By our assumptions, however, $Q_{d} \cap P_{b}=\varnothing$, and therefore there cannot be an edge between $b$ and $d$. As a result, since $T$ is a triangulation, there must be some vertices other than $a, b, c, d$. This takes care of the base of the induction. (As a warm-up, the reader may want to examine the case where $T$ has 5 vertices.)

Set $J=V-\{a, c, d\}$. Let $a=v_{0}, v_{1}, \ldots, v_{n}, v_{n+1}=c$ be the neighbors of $d$ in $T$ in clockwise order. Note that $b \notin\left\{v_{0}, v_{1}, \ldots, v_{n+1}\right\}$. If $Q_{v_{i}}$ and $P_{v_{i}}$ are incompatible for some $i=1,2, \ldots, n$, then we are done. So assume that $Q_{v_{i}}$ and $P_{v_{i}}$ are compatible for $i=1,2, \ldots, n$.

Our method of proof is to find a nonempty set of vertices $H \subset\left\{v_{1}, v_{2}\right.$, $\left.\ldots, v_{n}\right\}$ so that $Q_{H}$ is disjoint from $P_{J-H}$. After this is done, we examine the packings obtained by coalescing $Q_{H}$ into $Q_{d}$ and $P_{H}$ into $P_{d}$. Then the induction hypothesis establishes the theorem.

The proof is divided into three sections.

a. Constructing $H$. Let $E$ be the connected component of $D-\left(P_{J} \cup Q_{d}\right)$ whose boundary intersects $Q_{d}$. See Figure 3.4 on page 136. $P_{J}$ is disjoint from $Q_{d}$, and is connected. Therefore $E$ is well defined. We think of $E$ as a quadrilateral whose edges are, in clockwise order, $E_{a}=\partial E \cap D_{1}, E_{P}=\partial E \cap P_{J}$, $E_{c}=\partial E \cap D_{3}, E_{Q}=\partial E \cap Q_{d}$. Note that there is no loss of generality in assuming that $E_{Q}$ is a simple curve, because we can easily modify $Q_{d}$ slightly so that it has a nice boundary without disrupting the hypotheses of the theorem. Thus we assume that this is the case. We think of $E_{Q}$ and $E_{P}$ as being oriented from $D_{1}$ to $D_{3}$, with $E_{Q}$ having $E$ on its left, and $E_{P}$ having $E$ on its right.

With the intention of introducing some more notations, we walk along $E_{P}$. As we walk on $E_{P}$, we visit, in consecutive order, the sets $P_{v_{1}}, P_{v_{2}}, \ldots, P_{v_{n}}$. This gives a sequence of arcs $\gamma_{1}, \gamma_{2}, \ldots, \gamma_{n}$. In other words, $E_{P}$ consists of these arcs in this order, and each arc $\gamma_{i}$ lies on the boundary of $P_{v_{i}}$. Let $p_{i}$ be the terminal point of the arc $\gamma_{i}, i=1,2, \ldots, n$ (this is also the initial point of $\gamma_{i+1}$ for $\left.i<n\right)$, and let $p_{0}$ be the initial point of $\gamma_{1}$.

For $i=1,2, \ldots, n, v_{i}$ neighbors with $d$. Pick some arbitrary point $q_{i}$ in the intersection $Q_{v_{i}} \cap Q_{d}$. It is important to note that the points $q_{1}, q_{2}, \ldots, q_{n}$ appear in that order on $E_{Q} \cdot\left(E_{Q}\right.$ is oriented from $D_{1}$ to $D_{3}$.) Again, let $i$ be some index in the range $i=1,2, \ldots, n$. We say that $v_{i}$ is an invader 


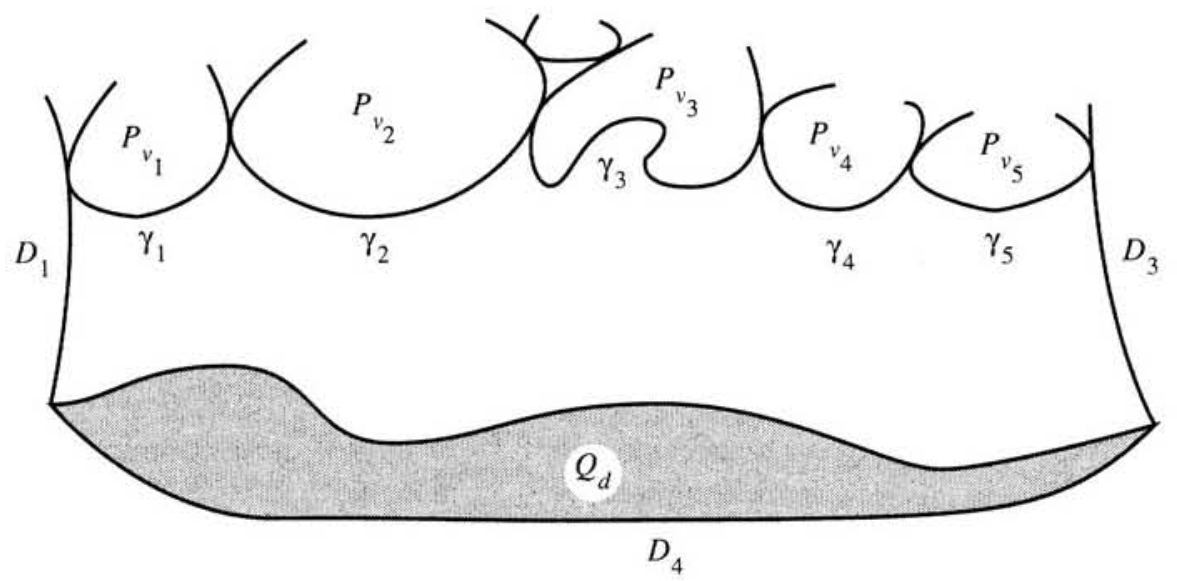

FIGURE 3.4. The quadrilateral $E$ and its accessories.

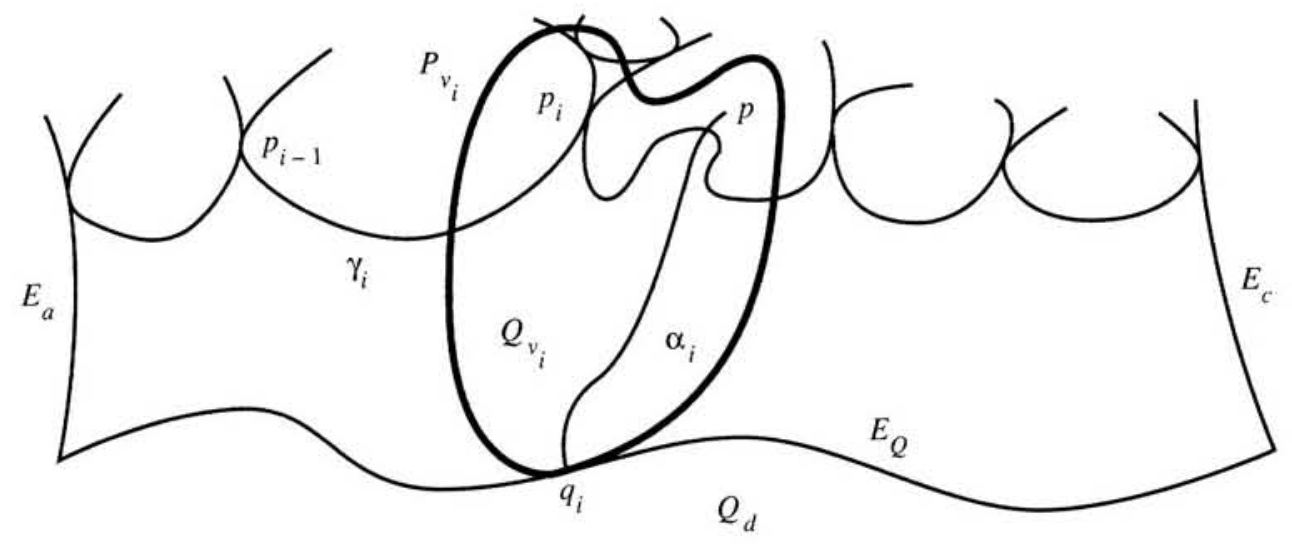

FIGURE 3.5. $v_{i}$ is an invader.

if $Q_{v_{i}}$ intersects $P_{J-\left\{v_{i}\right\}}-\left\{p_{i-1}, p_{i}\right\}$. See Figure 3.5. Consider some invader $v_{i}$ and some point $p \in Q_{v_{i}} \cap\left(P_{J-\left\{v_{i}\right\}}-\left\{p_{i-1}, p_{i}\right\}\right)$. As $q_{i}$ and $p$ are in $Q_{v_{i}}$ - interior $\left(P_{v_{i}}\right)$, there is some simple curve, say $\beta_{i}$, connecting them in interior $\left(Q_{v_{i}}-P_{v_{i}}\right)$, because $Q_{v_{i}}$ and $P_{v_{i}}$ are compatible. We orient $\beta_{i}$ from $q_{i}$ to $p$. Let $r$ be the first point of $\beta_{i}$ that is in $P_{J}$ ( $\beta_{i}$ has some point of $P_{J}$, namely $p$ ), and let $\alpha_{i}$ be the part of $\beta_{i}$ extending from $q_{i}$ to $r . \alpha_{i}$ is a cross-cut of the quadrilateral $E$. It has one endpoint, $q_{i}$, in $E_{Q}$ and one endpoint, $r$, in $E_{P}$.

Note that $r$, the endpoint of $\alpha_{i}$ that is on $E_{P}$, is not in $\gamma_{i}$. If $r \neq p$, then this is clear, because the curve $\beta_{i}$ avoids $P_{v_{i}}$, except perhaps at its endpoints. If $r=p$, then we know that $r \neq p_{i-1}, p_{i}$, and since $p \in P_{J-\left\{v_{i}\right\}}, p \notin \gamma_{i}$.

For every invader $v_{i}$ there are two possibilities. Either $\alpha_{i}$ separates $\gamma_{i}$ from $E_{a}$ in $E$, or it separates $\gamma_{i}$ from $E_{c}$ in $E$. (See Figure 3.5.) In the first case we call $i$ a left invader, and in the second case we call $i$ a right invader. Note that 


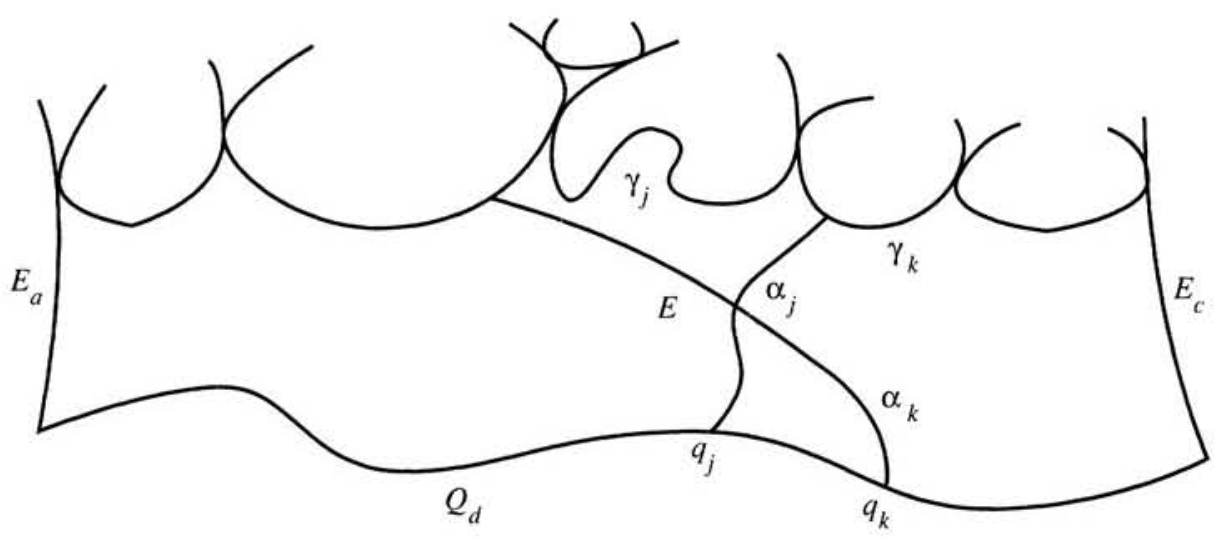

FIGURE 3.6. $\alpha_{j}$ and $\alpha_{k}$ must cross, but this is impossible.

if $i$ is a left invader, then $\alpha_{i}$ separates in $E$ every one of the points $q_{h}, h<i$ from every one of the arcs $\gamma_{m}, m \geqslant i$. Similarly, if $i$ is a right invader, then $\alpha_{i}$ separates in $E$ every one of the points $q_{h}, h>i$ from every one of the arcs $\gamma_{m}, m \leqslant i$.

For notational convenience, let 0 be considered a right invader, and let $n+1$ be considered a left invader. Since 0 is a right invader, $n+1$ is left invader, and every invader is either right or left, there will be some indices $0 \leqslant j<k \leqslant n+1$ so that $j$ is a right invader, $k$ is a left invader, and there is no invader between $j$ and $k$. (To find such $j, k$, start with $j=0, k=n+1$. If there is no invader between $j$ and $k$, stop. Otherwise pick some invader $i$ in the range $j<i<k$. If $i$ is a right invader, set $j:=i$. Otherwise, set $k:=i$. Continue in this manner until a situation is reached in which there is no invader $i, j<i<k$.) Suppose that $j<k$ are such indices, and let $H=\left\{v_{j+1}, v_{j+2}, \ldots, v_{k-1}\right\}$.

b. Showing that $H \neq \varnothing$, and that $Q_{H}$ is disjoint from $P_{J-H}$. To prove $H \neq \varnothing$, it is sufficient to demonstrate that $j+1 \neq k$. Wishing to arrive at a contradiction, assume that $j+1=k$. Since $n>0$, either $j>0$ or $k<n+1$ (or both). Because these cases are symmetric, we assume that $k<$ $n+1$. As $k$ is a left invader, it is obvious that $k>1$ and therefore, also $j=k-1>0$. Again, because $k$ is a left invader, $\alpha_{k}$ separates $q_{j}$ from $\gamma_{k}$ in $E$. Similarly, $\alpha_{j}$ separates $q_{k}$ from $\gamma_{j}$ in $E$. Since $\gamma_{j}$ comes before $\gamma_{k}$ on $E_{P}$ and $q_{j}$ comes before $q_{k}$ on $E_{Q}$, this implies that the curves $\alpha_{j}$ and $\alpha_{k}$ must cross. (See Figure 3.6.) This is impossible, because $v_{k} \neq v_{j}$, and $\operatorname{relint}\left(\alpha_{j}\right) \subset \operatorname{interior}\left(Q_{v_{j}}\right)$, relint $\left(\alpha_{k}\right) \subset \operatorname{interior}\left(Q_{v_{k}}\right)$. This contradiction shows that $k>j+1$, thus establishing $H \neq \varnothing$.

Now we will see that $Q_{H} \cap P_{J-H}=\varnothing$; i.e., that $Q_{v_{h}} \cap P_{J-H}=\varnothing$ for $j<$ $h<k$. Let $h$ be an index in the range $j<h<k$. If $Q_{v_{h}} \cap P_{J-\left\{v_{h}\right\}}=\varnothing$, then obviously $Q_{v_{h}} \cap P_{J-H}=\varnothing$. So assume otherwise, and let $p$ be a point in $Q_{v_{h}} \cap P_{J-\left\{v_{h}\right\}}$. We need to show that $p \notin P_{J-H}$. Because $v_{h}$ is not an invader, 
we know that $p$ must be $p_{h}$ or $p_{h-1}$. Since these cases are treated similarly, we only consider the case $p=p_{h}$.

The point $p_{h}$ is in the intersection $P_{v_{h}} \cap P_{v_{h+1}}$. Because the intersection of any three sets in the packing $P$ is empty, no other set $P_{u}\left(u \neq v_{h}, v_{h+1}\right)$ contains $p_{h}$. Thus we only need to worry about $P_{v_{h+1}}$. If $h+1<k$, then $v_{h+1} \in H$, and if $h+1=k=n+1$, then $v_{h+1}=c \notin J$. Therefore we may, and do consider only the case $h+1=k<n+1$. Now, $q_{h}, P_{h} \in Q_{v_{h}}-\operatorname{interior}\left(P_{v_{h}}\right)$, and $Q_{v_{h}}$ and $P_{v_{h}}$ are compatible. Therefore, there is some curve, say $\alpha$, that connects $q_{h}$ and $p_{h}$ in interior $\left(Q_{v_{h}}-P_{v_{h}}\right)$.

The curve $\alpha$ cannot cross $\alpha_{k}$, because interior $\left(Q_{v_{h}}\right)$ and $\operatorname{interior}\left(Q_{v_{k}}\right)$ are disjoint. Similarly, relint $(\alpha)$ is disjoint from $E_{Q}$. Also, $\operatorname{relint}(\alpha)$ is disjoint from $E_{P}$, because it certainly is disjoint from $\gamma_{h} \subset P_{v_{h}}$, and $v_{h}$ is not an invader. Thus relint $(\alpha) \subset E$. But the two endpoints of $\alpha, q_{h}$, and $p_{h}=p_{k-1}$ are separated in $E$ by $\alpha_{k}$, because $v_{k}$ is a left invader. This means that $\alpha$ must cross $\alpha_{k}$; a contradiction. This contradiction establishes $Q_{v_{h}} \cap P_{J-H}=\varnothing$, and therefore $Q_{H} \cap P_{J-H}=\varnothing$.

c. Applying the inductive hypothesis. Let $Q^{\prime}$ and $P^{\prime}$ be the packings obtained from $Q$ and $P$, respectively, by coalescing $H \cup\{d\}$ while keeping $b$. Let $T^{\prime}=T^{\prime}\left(V^{\prime}\right)$ be the triangulation that is the common nerve of these two packings, and let $d^{\prime}$ be the vertex of $T^{\prime}$ corresponding to the coalesced set $H \cup\{d\}$. Because of part b, we have $Q_{d^{\prime}}^{\prime} \cap P_{V^{\prime}-\left\{a, c, d^{\prime}\right\}}^{\prime}=\varnothing$, provided that the touch-up modification done in the coalescing procedure is not too big. The inductive hypothesis now applies to the packings $Q^{\prime}$ and $P^{\prime}$, and establishes the theorem.

\section{RIGIDITY OF INFINITE CIRCLE PACKINGS THAT ALMOST FILL THE SPHERE}

In this section we prove Theorem 1.1.

4.1. Definitions. Let $P=\left(P_{v}: v \in V\right)$ be a finite or infinite packing in $S^{2}$. An interstice of $P$ is a connected component of the complement of $P_{V}=\bigcup_{v \in V} P_{v}$ whose boundary is formed by finitely many of the sets $P_{v}$. The carrier of $P$, carrier $(P)$, is the union of all the interstices and all the sets $P_{v}$. A connected component of $S^{2}-\operatorname{carrier}(P)$ is called a singularity of $P$. A singularity is parabolic if it consists of a single point. And singular $(P)$ denotes the union of the singularities of $P$ : singular $(P)=S^{2}-\operatorname{carrier}(P)$.

Proof of Rigidity Theorem 1.1. Let $P=\left(P_{v}: v \in V\right)$ be another circle packing whose nerve is $T$, and let $[a, b, c]$ be some triangle in $T$. By making an appropriate initial normalization, we assume that $P_{v}=Q_{v}$ for $v=a, b, c$. (The Möbius transformation that takes the three intersection points of the circles $P_{a}, P_{b}, P_{c}$ to the corresponding intersection points of $Q_{a}, Q_{b}, Q_{c}$ also takes $P_{a}, P_{b}, P_{c}$ to $Q_{a}, Q_{b}, Q_{c}$, respectively. 
If $Q_{v}=P_{v}$ for each $v \in V$, then we are done. With the intention of reaching a contradiction, we assume that this is not the case. Starting from $[a, b, c]$, we walk on triangles of $T$ by moving from a triangle to an adjacent triangle. We can do this, and reach some first triangle where the three circles corresponding to the vertices are not the same in both packings. So, without loss of generality, we assume that $Q_{d} \neq P_{d}$, where $d$ is the vertex other than $b$ that makes a triangle with $a$ and $c$. We further assume that the point $\infty$ is contained in the interstice corresponding to the triangle $[a, b, c]$. Thus our packings lie in the plane.

There are two possibilities. Either $Q_{d}$ is smaller than $P_{d}$, and the situation is as in Figure 4.1(a) on the next page, or the other way around. Both cases are treated similarly, and we assume that the situation is as in Figure 4.1(a). Let $p$ be the point of intersection of $P_{a}$ and $P_{c}$, which is also the point of intersection of $Q_{a}$ and $Q_{c}$. Pick some number $\beta>1$ with $\beta-1$ small, and expand the packing $Q$ by a homothety with center $p$ and expanding ratio $\beta$. Continue to denote the resulting packing by $Q$. The modified picture is given in Figure 4.1(b).

The boundary of $P_{V}=\bigcup_{v \in V} P_{v}$ is also the boundary of $\bigcup_{v \in V}$ interior $\left(P_{v}\right)$. Therefore it is nowhere dense, and thus is of Baire category I. Because singular $(Q)$ is countable, the set of translations $S$ for which $\operatorname{singular}(S(Q))$ intersects $\partial P_{V}$ is also of Baire category I. By Baire's theorem, this shows that there is an arbitrarily small translation $S$ for which $\operatorname{singular}(S(Q))$ is disjoint from $\partial P_{V}$. Apply such a translation to $Q$, and make it small enough so that qualitatively, Figure 4.1(b) is still correct, except in a small neighborhood of $p$. We continue to denote the resulting packing by $Q$.

The complement of $Q_{a} \cup Q_{b} \cup Q_{c} \cup Q_{d}$ consists of a quadrilateral that contains the other sets of the packing $Q$, and two trilaterals (one of the trilaterals contains infinity). Let $D_{Q}$ be the (closure of the) quadrilateral region, and let $Q_{a}^{\prime}, Q_{b}^{\prime}, Q_{c}^{\prime}, Q_{d}^{\prime}$ be the arcs of $D_{Q}$ that are on $Q_{a}, Q_{b}, Q_{c}, Q_{d}$, respectively. We have $D_{Q}=\left(Q_{a}^{\prime}, Q_{b}^{\prime}, Q_{c}^{\prime}, Q_{d}^{\prime}\right)$. Let the quadrilateral $D_{P}=\left(P_{a}^{\prime}, P_{b}^{\prime}, P_{c}^{\prime}, P_{d}^{\prime}\right)$ in $S^{2}-\left(P_{b} \cup P_{b} \cup P_{c} \cup P_{d}\right)$ be defined in the same manner. These two quadrilaterals are indicated in Figure 4.2. Define new packings $Q^{\prime}=\left(Q_{v}^{\prime}: v \in V\right)$ and $P^{\prime}=\left(P_{v}^{\prime}: v \in V\right)$, by $Q_{v}^{\prime}=Q_{v}, P_{v}^{\prime}=P_{v}$ for $v \neq a, b, c, d$, and $P_{v}^{\prime}, Q_{v}^{\prime}$ as defined above for $v=a, b, c, d$.

Our two quadrilaterals $D_{Q}, D_{P}$, and the two packings $Q^{\prime}, P^{\prime}$ are in the right relative position to apply the Incompatibility Theorem. (See Remark 3.2.) If we could apply the theorem to them, then we could conclude that there is some $v \in V-\{a, b, c, d\}$ so that $Q_{v}^{\prime}$ and $P_{v}^{\prime}$ are not compatible. This is impossible, because these sets are circles, and this contradiction would finish the proof. The problem is that these packings are not finite. We overcome this problem by using the fact that $\operatorname{singular}\left(Q^{\prime}\right)$ is disjoint from $\partial P_{V}^{\prime}$. We use this fact to cook up finite packings from $Q^{\prime}$ and $P^{\prime}$.

Let $\mathfrak{H}$ be the collection of all interstices of the packing $P^{\prime}$, and let $\mathfrak{L}$ be the collection of all the singularities of the packing $P^{\prime}$. The collection 


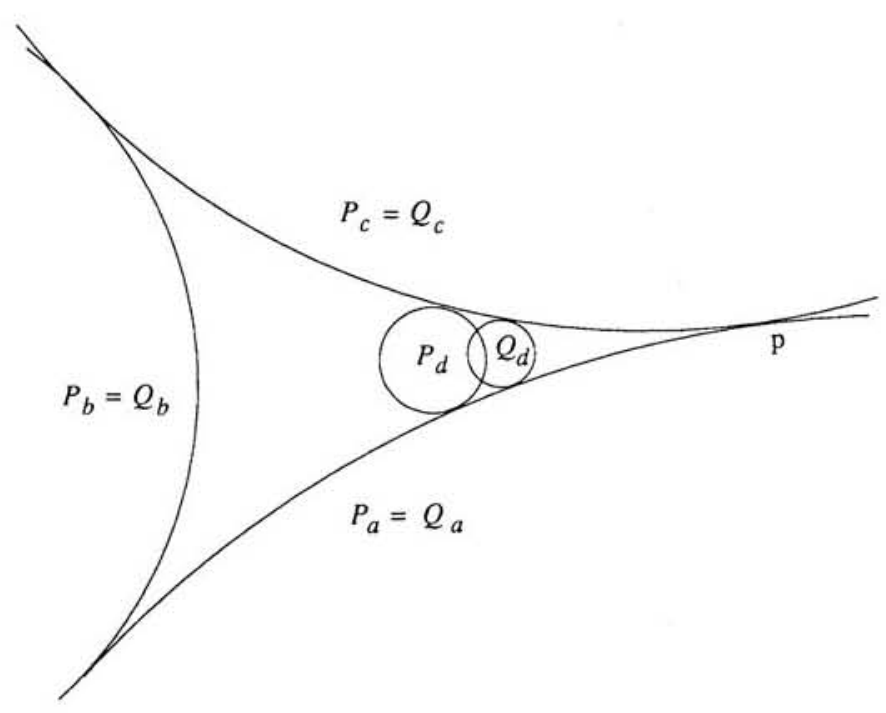

(a) Case $Q_{d}$ is smaller than $P_{d}$.

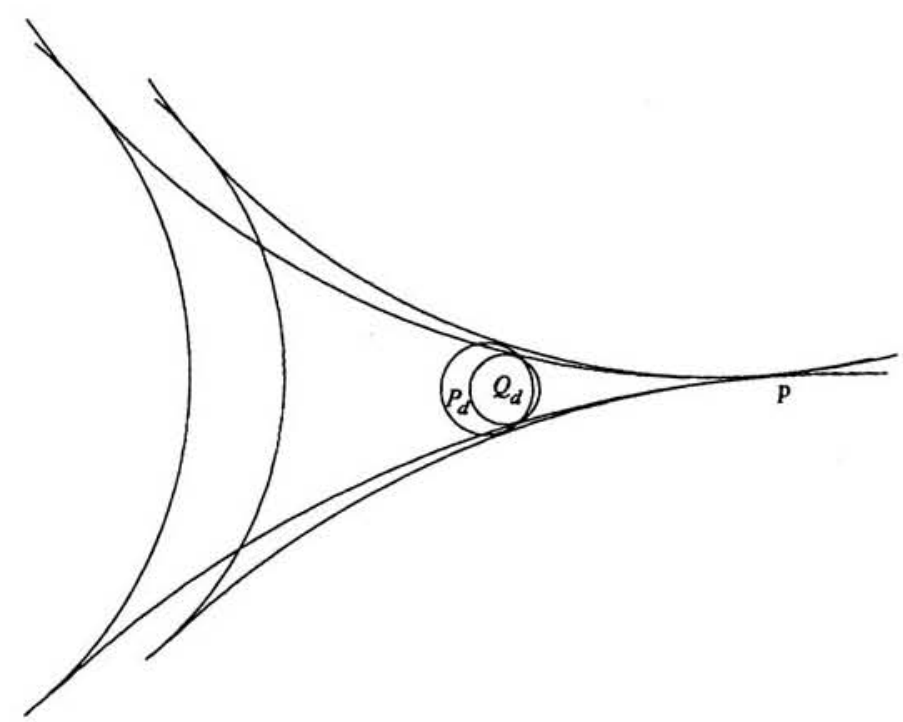

(b) After expanding $Q$.

FIGURE 4.1

$\left\{H\right.$, interior $\left(P_{v}^{\prime}\right)$, interior $\left.(L): H \in \mathfrak{H}, v \in V, L \in \mathfrak{L}\right\}$ is an open cover for the compact set $\operatorname{singular}\left(Q^{\prime}\right)$, because $\operatorname{singular}\left(Q^{\prime}\right) \cap \partial P_{V}^{\prime}=\varnothing$. Let $\left\{H\right.$, interior $\left(P_{v}^{\prime}\right)$, interior $\left.(L): H \in \mathfrak{H}^{\prime}, v \in V^{\prime}, L \in \mathfrak{L}^{\prime}\right\}$ be a finite sub- 


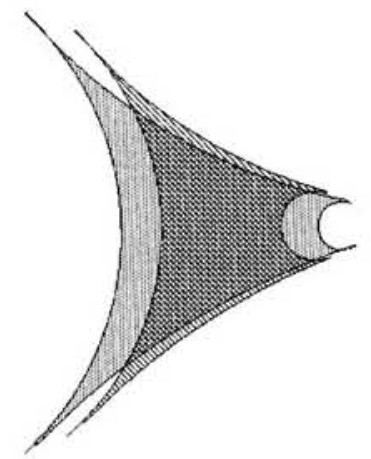

FIGURE 4.2. The quadrilaterals $D_{Q}$ and $D_{P}$.

cover; that is, $\mathfrak{H}^{\prime} \subset \mathfrak{H}, V^{\prime} \subset V, \mathfrak{L}^{\prime} \subset \mathfrak{L}$ are finite subsets with

$$
G \stackrel{\text { def }}{=}\left(\bigcup_{v \in V^{\prime}} \text { interior }\left(P_{v}^{\prime}\right)\right) \cup\left(\bigcup_{H \in \mathcal{S}^{\prime}} H\right) \cup\left(\bigcup_{L \in \mathcal{L}^{\prime}} \text { interior }(L)\right),
$$

containing $\operatorname{singular}\left(Q^{\prime}\right)$. Let $V_{1}$ be the set of vertices $v \in V$ so that $Q_{v}^{\prime}$ is not contained in $G . V_{1}$ is a finite set, because for any infinite sequence of distinct circles in the packing $Q^{\prime}$, the radii of the circles must tend to zero, and any accumulation point for the sequence is necessarily in $\operatorname{singular}\left(Q^{\prime}\right)$, which is a compact subset of the open set $G$.

Let $V_{2}$ be some finite connected set of vertices that contains $V_{1} \cup\{a, b, c, d\}$, and let $\hat{P}$ and $\hat{Q}$ be the packings obtained from $P^{\prime}$ and $Q^{\prime}$, respectively, by coalescing each connected component of $V-V_{2}$ while keeping $a$. (Recall the technique of coalescing from 3.3.) Because $\hat{P}$ and $\hat{Q}$ are finite, we can now apply the incompatibility theorem. We conclude from it that there are sets $\hat{P}_{w}$ and $\hat{Q}_{w}$, both corresponding to the same vertex $w \neq a, b, c, d$ that are not compatible. This vertex $w$ cannot be in $V_{2}$, because the sets of $\hat{P}$ and $\hat{Q}$ that correspond to vertices in $V_{2}$ are circles, and circles are always compatible.

Let $W$ be the connected component of $V-V_{2}$ that coalesced to form $w$. The union $Q_{W}^{\prime}=\bigcup_{v \in W} Q_{v}^{\prime}$ is a connected set whose closure is contained in $G$. The union defining $G$ is a finite union of disjoint open sets. Because $Q_{W}^{\prime}$ is connected, $\overline{Q_{W}^{\prime}}$ is contained in one set of this union, say $\overline{Q_{W}^{\prime}} \subset$ interior $(F)$, with $F \in \mathfrak{H}^{\prime} \cup \mathfrak{L}^{\prime}$ or $F=P_{u}^{\prime}$ for some $u \in V^{\prime}$. If $F$ is not disjoint from $P_{W}$, then $F$ is contained in $P_{W}$. Thus $\overline{Q_{W}^{\prime}}$ is either contained in the interior of $P_{W}$, or is disjoint from $P_{W} . \hat{P}_{w}$ and $\hat{Q}_{w}$ are compatible, therefore, provided that the modifications done during the coalescing procedure are small enough. This contradicts our previous conclusion, and thereby completes the proof of the theorem.

It is possible to obtain a generalization of this theorem to packings by convex sets. This is given at the end of the next section. 


\section{RIGIDITY OF PACKINGS THAT ALMOST FILL A DISK}

5.1. Rigidity Theorem, hyperbolic case. Let $T=T(V)$ be an infinite, planar triangulation. Let $Q=\left(Q_{v}: v \in V\right)$ be a circle packing in the open unit disk $U$ with nerve $T$, and suppose that $U-\operatorname{carrier}(Q)$ is at most countable. Let $P$ be another circle packing in $U$ with nerve $T$. Further assume that the singularity of $P$ that corresponds to the singularity $S^{2}-U$ of $Q$ is also equal to $S^{2}-U$. Then $Q$ and $P$ are Möbius equivalent.

We need the following lemma, which is topological in nature.

5.2. Lemma. Let $T=T(V)$ be a finite triangulation of the sphere, and let $a \leftrightarrow b$ be an edge in $T$. Suppose that $P=\left(P_{v}: v \in V\right)$ and $Q=\left(Q_{v}: v \in V\right)$ are two nondegenerate packings of topological disks on $S^{2}$ with nerve $T$. Further suppose that $P_{a} \subset$ interior $\left(Q_{a}\right) ; Q_{b} \subset$ interior $\left(P_{b}\right)$; and $Q_{z} \subset$ interior $\left(P_{z}\right)$ [or $P_{z} \subset$ interior $\left.\left(Q_{z}\right)\right]$ for some $z \neq a, b$ in $V$. Then $Q_{v}$ and $P_{v}$ are incompatible for some vertex $v \in V-\{a, b, z\}$.

Proof. The idea is to take a double cover, and obtain a situation to which the Incompatibility Theorem can be applied. The statement of the lemma is symmetric in $Q$ and $P$, and thus we consider only the case $Q_{z} \subset$ interior $\left(P_{z}\right)$.

Pick some points $s_{a} \in$ interior $\left(P_{a}\right)$ and $s_{z} \in \operatorname{interior}\left(Q_{z}\right)$. Consider a double cover $M$ of $S^{2}$, in the topological sense, branched over $s_{a}$ and $s_{z}$. The Packings $P, Q$ lift to packings $P^{\prime}=\left(P_{v}^{\prime}: v \in V^{\prime}\right), Q^{\prime}=\left(Q_{v}^{\prime}: v \in V^{\prime}\right)$ in $M$, where to each vertex $v \in V-\{a, z\}$ correspond two vertices in $V^{\prime}$, say $v_{1}, v_{2}$, and to each of $a$ and $z$ corresponds one vertex in $V^{\prime}$, say $a^{\prime}$ and $z^{\prime}$, respectively. Figure 5.1(a) illustrates the sets corresponding to the vertices $a$ and $b$ downstairs, and Figure 5.1(b) illustrates those corresponding to $a^{\prime}, b_{1}, b_{2}$ in the double cover $M$.

For convenience, we assume that $P_{a} \cap P_{b}$ contains precisely two distinct points, say $p, p^{\prime}$, and that similarly $Q_{a} \cap Q_{b}=\left\{q, q^{\prime}\right\}$. There clearly is no loss of generality in this assumption, because we can make slight modifications to $P_{a}$ near $P_{b}$ and to $Q_{a}$ near $Q_{b}$. Let $p_{1}, p_{2}, p_{1}^{\prime}, p_{2}^{\prime}, q_{1}, q_{2}, q_{1}^{\prime}, q_{2}^{\prime}$ be the corresponding points in the double cover $M$. Let $D_{Q}$ be the closure of the connected component of $M-\left(Q_{b_{1}}^{\prime} \cup Q_{a^{\prime}}^{\prime} \cup Q_{b_{2}}^{\prime}\right)$ that contains the other sets of $Q^{\prime}$, and let $D_{P}$ be the closure of the connected component of $M-\left(P_{b_{1}}^{\prime} \cup P_{a^{\prime}}^{\prime} \cup P_{b_{2}^{\prime}}\right)$ that contains the other sets of $P^{\prime}$. We view $D_{Q}$ and $D_{P}$ as quadrilaterals with vertices $q_{1}, q_{2}, q_{1}^{\prime}, q_{2}^{\prime}$ and $p_{1}, p_{2}, p_{1}^{\prime}, p_{2}^{\prime}$, respectively. Let $P_{a_{1}}^{\prime \prime}$ and $P_{a_{2}}^{\prime \prime}$ be the two edges of the quadrilateral $D_{P}$ that are on the boundary of $P_{a^{\prime}}^{\prime}$. Let $P_{b_{1}}^{\prime \prime}$ and $P_{b_{2}}^{\prime \prime}$ be the two edges of $D_{P}$ that are on $P_{b_{1}}^{\prime}$ and $P_{b_{2}}^{\prime}$, respectively. Similarly define the edges of $D_{Q}, Q_{a_{1}}^{\prime \prime}, Q_{a_{2}}^{\prime \prime}, Q_{b_{1}}^{\prime \prime}, Q_{b_{2}}^{\prime \prime}$. See Figure 5.2.

We now define modified packings $P^{\prime \prime}=\left(P_{v}^{\prime \prime}: v \in V^{\prime \prime}\right), Q^{\prime \prime}=\left(Q_{v}^{\prime \prime}: v \in V^{\prime \prime}\right)$. Set $V^{\prime \prime}=\left(V^{\prime} \cup\left\{a_{1}, a_{2}\right\}\right)-\left\{a^{\prime}\right\} . P_{v}^{\prime \prime}$ and $Q_{v}^{\prime \prime}$ have been defined above for $v=a_{1}, a_{2}, b_{1}, b_{2}$. Set $P_{v}^{\prime \prime}=P_{v}^{\prime}$ and $Q_{v}^{\prime \prime}=Q_{v}^{\prime}$ for other $v \in V^{\prime \prime}$. The 


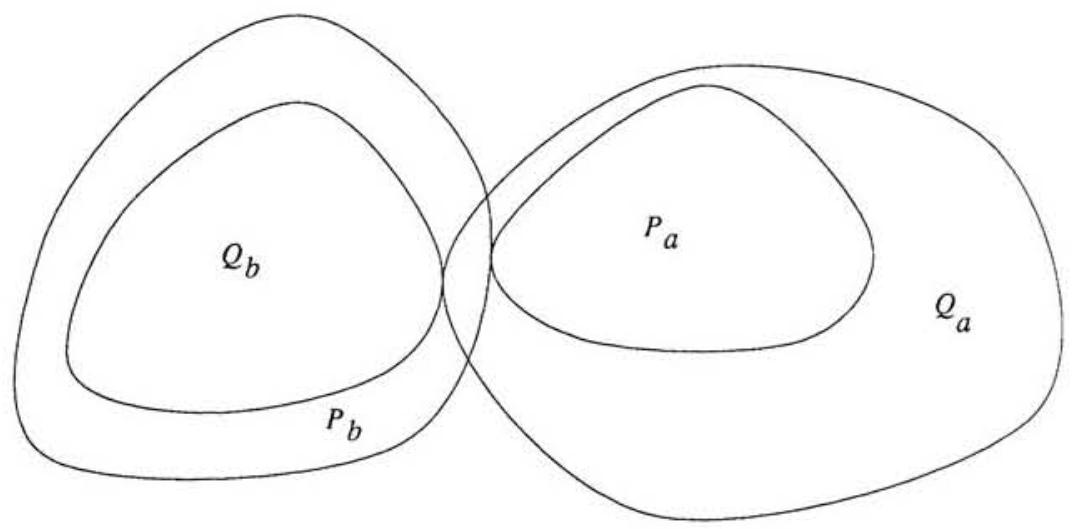

Figure 5.1(a). The sets corresponding to $a$ and $b$.

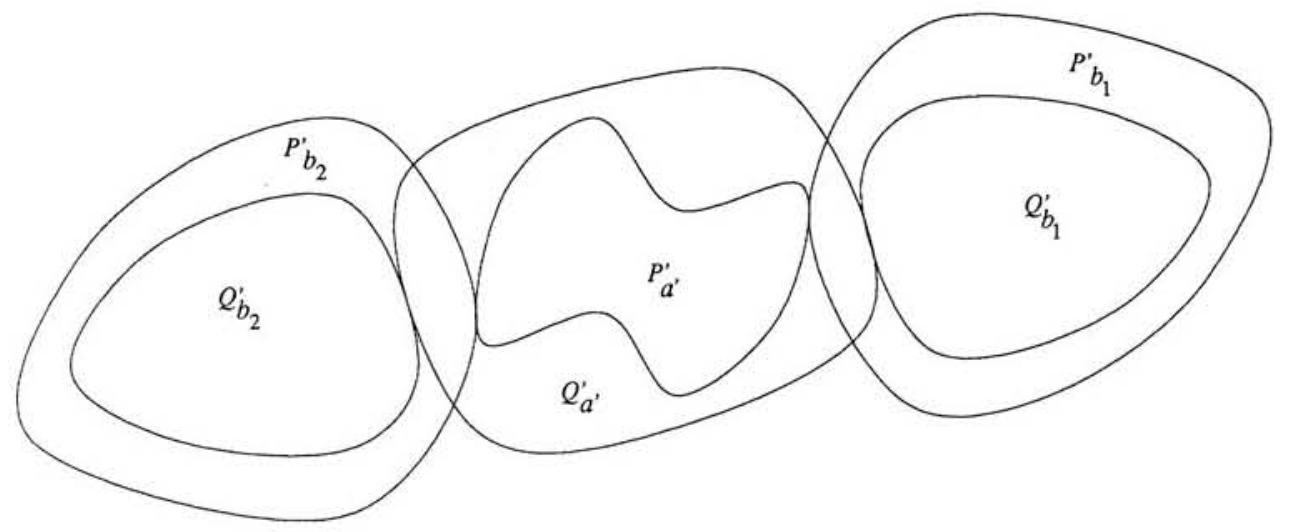

FIGURE 5.1(b). Sets corresponding to $a^{\prime}, b_{1}, b_{2}$ in $M$.

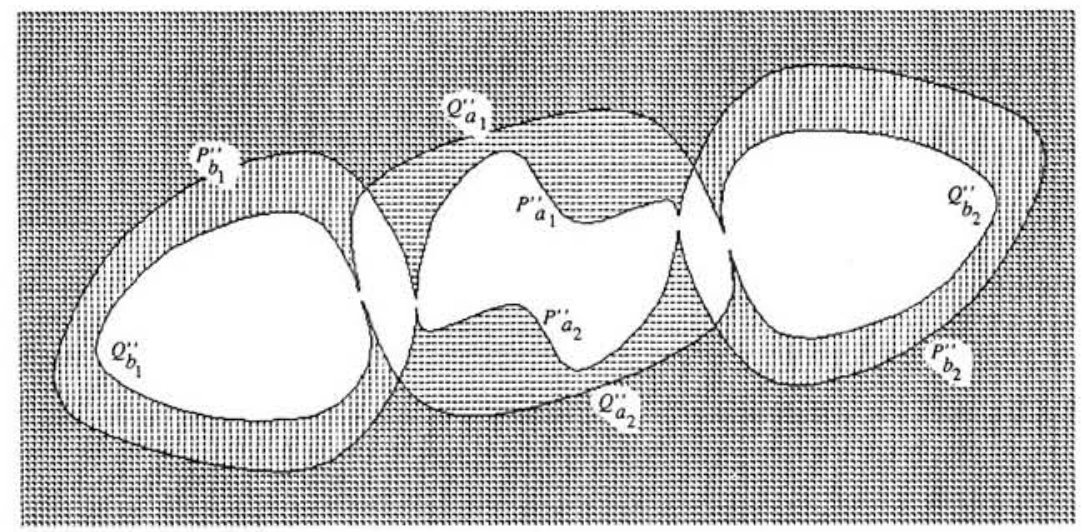

Figure 5.2. The quadrilaterals $D_{P}$ and $D_{Q}$. 


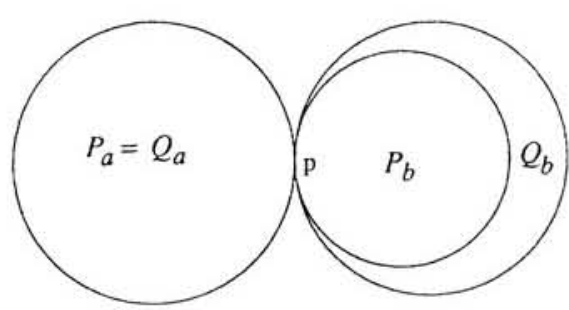

(a) Case $P_{b} \varsubsetneqq Q_{b}$.

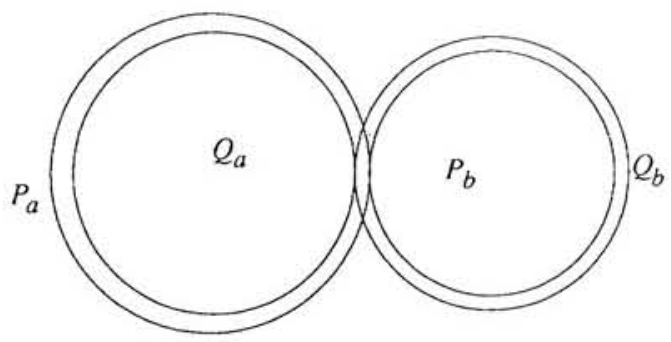

(b) After expanding and translating $P$.

FIGURE 5.3

packings $Q^{\prime \prime}, P^{\prime \prime}$ have as nerve a common triangulation $T^{\prime \prime}$ of a quadrilateral with boundary $a_{1}, a_{2}, b_{1}, b_{2}$ (provided the labeling in the branching $v \rightarrow$ $v_{1}, v_{2}$ is done consistently for both packings), which can roughly be described as a double cover of $T$ branched over the vertex $z$ and the edge $a \leftrightarrow b$. It is clear that the incompatibility theorem applies to these packings and gives a vertex $w \in V^{\prime \prime}-\left\{a_{1}, a_{2}, b_{1}, b_{2}\right\}$ for which $Q_{w}^{\prime \prime}$ and $P_{w}^{\prime \prime}$ are incompatible. It is impossible that $w=z^{\prime}$, because $Q_{z^{\prime}}^{\prime \prime} \subset$ interior $\left(P_{z^{\prime}}^{\prime \prime}\right)$. Thus $w=v_{j}$, say, with $v \in V-\{a, b, z\}$, and $j=1$ or $j=2$. But this implies that $Q_{v}$ and $P_{v}$ are incompatible, as needed.

Proof of 5.1. After a few initial normalizations, we reach a situation where the above lemma can be applied.

Let $Q_{s}$ be the singularity $S^{2}-U$ of the packing $Q$, and let $P_{s}$ be the corresponding singularity of $P$. (We assume that the symbol $s$ is not a vertex of $T$.)

Pick some edge $a \leftrightarrow b$ in $T$, and let $p$ be the point of intersection of $Q_{a}$ and $Q_{b}$. After renormalizing by a Möbius transformation taking $U$ onto $U$ (i.e., a hyperbolic isometry), we may, and do, also assume that $P_{a} \cap P_{b}=\{p\}$, and that the outward unit normal of $Q_{a}$ at $p$ is the same as that of $P_{a}$.

There is some (unique) $\alpha>0$ so that expanding $P_{a}$ by a homothety with center $p$ and expansion $\alpha$ takes $P_{a}$ to $Q_{a}$. Apply this expansion to the packing $P$, and continue to denote the resulting packing by $P$. Now $P_{s}$ is either contained in $Q_{s}$, or contains $Q_{s}$, depending on whether $\alpha \geqslant 1$ or $\alpha \leqslant 1$, respectively.

Case 1. $P_{b} \varsubsetneqq Q_{b}$. See Figure 5.3(a). Expand the packing $P$ by a homothety with center $p$. Make the expansion ratio $\beta>1$, but small enough so that afterwards $P_{a} \supsetneqq Q_{a}, P_{b} \varsubsetneqq Q_{b}$, and $P_{s} \subset$ interior $\left(Q_{s}\right)$ or $Q_{s} \subset$ interior $\left(P_{s}\right)$. $\left(P_{s} \subset\right.$ interior $\left(Q_{s}\right)$ would be the case, if $\alpha \geqslant 1$, and $Q_{s} \subset$ interior $\left(P_{s}\right)$, otherwise.) Now translate the packing $P$ slightly in the direction of the outward normal to $P_{a}$ and $Q_{a}$ at $p$. After the translation, we have $Q_{a} \subset$ interior $\left(P_{a}\right)$, $P_{b} \subset$ interior $\left(Q_{b}\right)$, and $P_{s} \subset$ interior $\left(Q_{s}\right)$ or $Q_{s} \subset$ interior $\left(P_{s}\right)$. See Figure 5.3(b). Finally, make a small perturbing translation that does not destroy the 
above relations, but makes all the singularities of $Q$, except possibly $Q_{s}$, be disjoint from the boundary of $P_{V}=\bigcup_{v \in V} P_{v}$. By Baire's category theorem, this can be done, as in the proof of 1.1.

We assume that $P_{s} \subset$ interior $\left(Q_{s}\right)$. The other possibility is dealt with similarly. Let $Z$ be a set of vertices whose boundary is finite, so that all the sets $P_{v}, v \in Z$ are contained in interior $\left(Q_{s}\right)$, and $P_{V-Z}$ is bounded away from $P_{s}$. (To see that one can find such a set of vertices, let $V_{n}$ be the set of vertices whose distance from $a$ is at most $n$. Let $C_{n}$ be the simple closed path in the boundary of $V_{n}$ so that $P_{C_{n}}$ separates $P_{V_{n}-C_{n}}$ from $P_{s}$. As $n \rightarrow \infty$, $P_{C_{n}} \rightarrow \partial P_{s}$. Thus, for sufficiently large $n$, one can take $Z$ to be the connected component of $V-C_{n}$ that does not contain $a$.)

Let $P^{\prime}$ and $Q^{\prime}$ be the packings obtained by coalescing $Z$ to some vertex, say $z$, while keeping $a$. We make further coalescings, as in the proof of 1.1, but keep the vertices $a, b, z$. Then to each set in these packings we adjoin the connected components of its complement which do not intersect $Q_{a}$ (to make them into topological disks). Eventually, we obtain finite packings $P^{\prime \prime}, Q^{\prime \prime}$ with a common triangulation as nerve; corresponding sets being compatible; and $P_{z}^{\prime \prime} \subset$ interior $\left(Q_{z}^{\prime \prime}\right), Q_{a}^{\prime \prime} \subset$ interior $\left(P_{a}^{\prime \prime}\right)$, and $P_{b}^{\prime \prime} \subset$ interior $\left(Q_{b}^{\prime \prime}\right)$. This contradicts the lemma, and thus Case 1 is ruled out.

Case 2. $Q_{b} \varsubsetneqq P_{b}$. We make the same argument in this case, only now take $0<\beta<1$ and interchange the roles of $a$ and $b$.

Case 3. $Q_{b} \not \subset P_{b}$ and $P_{b} \not \subset Q_{b}$. This case is impossible, because the boundaries of $P_{b}$ and $Q_{b}$ both pass through $p$ and have the same unit outward normal there.

Case 4. $Q_{b}=P_{b}$. If $Q_{v}=P_{v}$ for all $v \in V$, then we are done, so assume otherwise. We also have $Q_{a}=P_{a}$, and therefore there is some triangle [c, $\left.d, e\right]$ in $T$ with $Q_{c}=P_{c}, Q_{d}=P_{d}$, and $Q_{e} \neq P_{e}$. We then have either $Q_{e}$ bigger than $P_{e}$ and $Q_{e} \cup Q_{c} \cup Q_{d}$ separates $P_{e}$ from every $P_{v}, v \neq c, d, e$, as in Figure 5.4(a) (see p. 146), or the other way around. Both situations are dealt with similarly, so assume that $Q_{e}$ is bigger than $P_{e}$. Let $p^{\prime}$ be the point of intersection of $P_{c}$ and $P_{d}$ (also $Q_{c} \cap Q_{d}=\left\{p^{\prime}\right\}$ ). Expand the packing $P$ by a homothety with center $p^{\prime}$ and expansion ratio $\beta>1$ with $\beta-1$ small. Then we have the sets $Q_{v}, P_{v}, v=c, d, e$ as in Figure 5.4(b), and $P_{s} \subset$ interior $\left(Q_{s}\right)$, if $\alpha \geqslant 1$, or $Q_{s} \subset$ interior $\left(P_{s}\right)$, otherwise. Now, if necessary, modify $Q_{e}$ so that its interior would contain $P_{e}$, as in Figure 5.4(c), while keeping $Q$ as a packing with the same combinatorics. (That is, $Q_{e}$ must still touch the sets it used to touch. It may perhaps no longer be a circle, though.) A very slight translation would now give $Q_{d} \subset$ interior $\left(P_{d}\right)$, and a contradiction can be reached as in case 1 , with $d$ replacing $a$ and $e$ replacing $b$.

In the proof above, except for the first normalization, all the transformations applied were translations and positive homotheties. Going through the proof, one sees that (except for this initial normalization), we did not use the fact that 


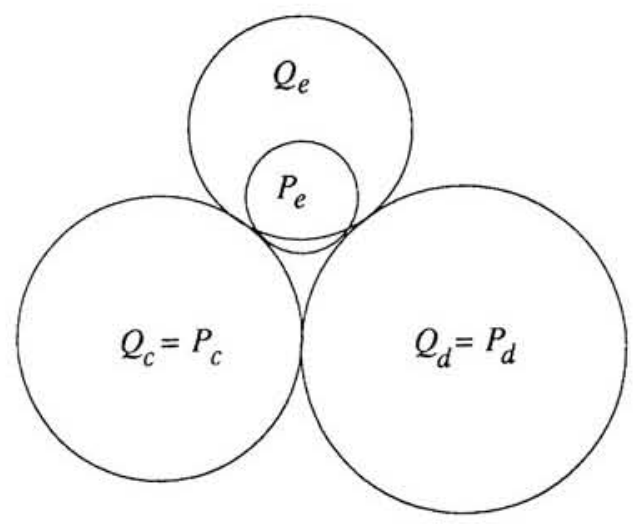

(a) Case 4, with $Q_{e}$ bigger than $P_{e}$.

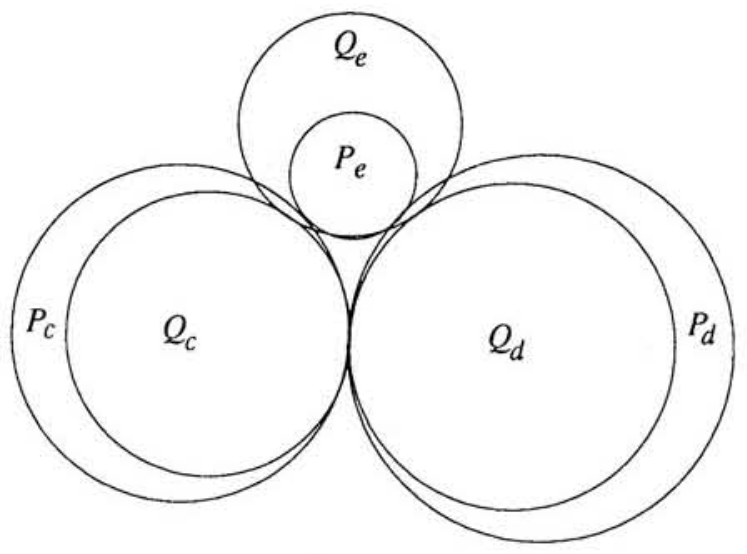

(b) After expanding $P$.

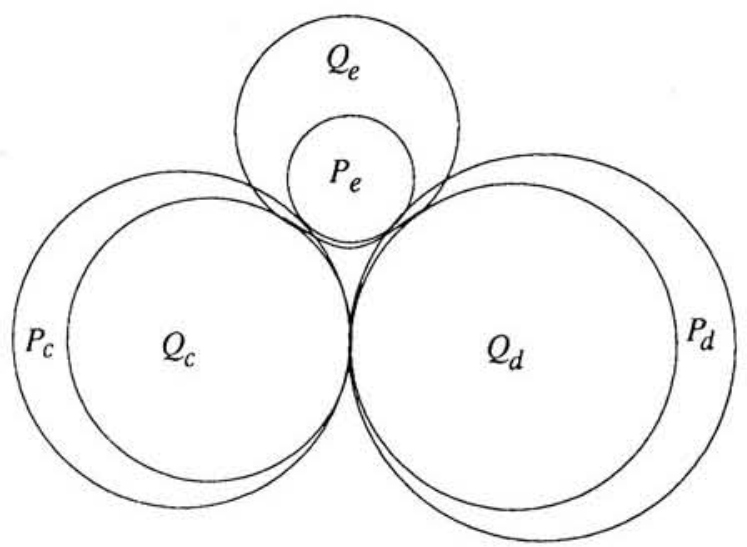

(c) After modifying $Q_{e}$.

FIGURE 5.4 


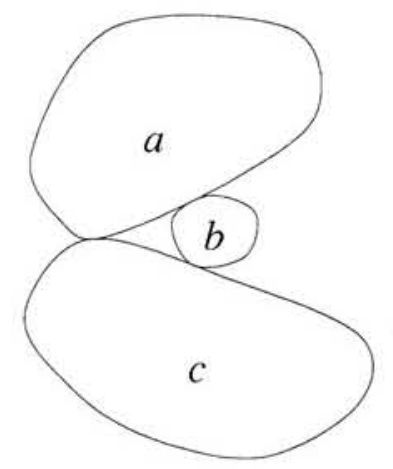

(a) Cannot find a circumscribing convex $U$.

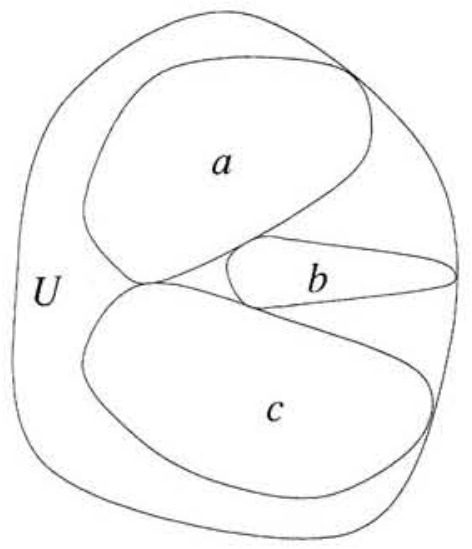

(b) After a harmless modification, such a $U$ exists.

FIGURE 5.5

we are working with circles and, in fact, strict convexity and smoothness are enough. Thus we have the following generalization of 5.1.

5.3. Theorem. Let $T=T(V)$ be an infinite, planar triangulation. Let $Q=$ $\left(Q_{v}: v \in V\right)$ be a packing with nerve $T$ of smooth strictly convex sets in an open convex set $U$. Suppose that $U-\operatorname{carrier}(Q)$ is at most countable. Then $Q$ is rigid up to three degrees of freedom, in the following sense. Let $a \leftrightarrow b$ be some edge in $T$, and let $P$ be another packing in $U$ with nerve $T$. Further assume that $P_{v}$ is positively homothetic to $Q_{v}$ for $v \in V$; the singularity of $P$ that corresponds to the singularity $S^{2}-U$ of $Q$ is also equal to $S^{2}-U$; $P_{a} \cap P_{b}=Q_{a} \cap Q_{b}$; and the outward unit normal of $P_{a}$ at this intersection point is the same as that of $Q_{a}$. Then $P=Q$.

The only need for smoothness is to insure that the packings be nondegenerate. One could dispense with the smoothness hypothesis, if one restricts the word 'packing' to mean 'nondegenerate packing'.

We also prove the following theorem, which can be seen as a generalization of 1.1 .

5.4. Theorem. Let $T=T(V)$ be a (possibly infinite) planar triangulation. Let $Q=\left(Q_{v}: v \in V\right)$ be a packing with nerve $T$ of smooth strictly convex bodies in the plane. Suppose that $\infty$ is contained in one of the interstices of $Q$, say in the interstice corresponding to the triangle $[a, b, c]$ of $T$. Further suppose that singular $(Q)$ is at most countable. Then $Q$ is rigid, in the following sense. Let $P$ be another packing in the plane with nerve $T ; P_{v}$ positively homothetic to $Q_{v}$ for each $v$; and $P_{v}=Q_{v}$ for $v=a, b, c$. Then $Q=P$.

Proof. First assume that there is some smooth convex body $U$ that contains $Q_{a} \cup Q_{b} \cup Q_{c}$, and each of $Q_{v}, v=a, b, c$, intersects the boundary of $U$. Let $T^{\prime}$ be the triangulation obtained from $T$ by adding one additional vertex, say $s$, 
in the triangle $[a, b, c]$, adjoining edges from $s$ to $a, b, c$, and splitting the triangle $[a, b, c]$ into three smaller triangles: $[a, b, s],[b, c, s],[c, a, s]$. Let $P^{\prime}$ and $Q^{\prime}$ be the packings obtained from $P$ and $Q$ by adding another set $Q_{s}^{\prime}=P_{s}^{\prime}=S^{2}-$ interior $(U)$. These packings obviously have nerve $T^{\prime}$.

Now, the proof of 5.1 Case 4 can be applied here, because the fact that $Q_{s}^{\prime}=P_{s}^{\prime}$ is not a singularity, but a packed set, only makes things easier. We get $P^{\prime}=Q^{\prime}$, which gives $P=Q$.

There may be a situation where no such set $U$ exists, as in Figure 5.5(a). However, we are free to manipulate the sets $P_{v}, Q_{v}, v=a, b, c$, provided we do not modify the parts of their boundaries that bound the component of $S^{2}-\left(Q_{a} \cup Q_{b} \cup Q_{c}\right)$ that contains the other sets of the packings. Thus we easily reduce the situation to the case where such a $U$ exists. See Figure 5.5(b).

\section{SOME PROBLEMS}

One is naturally led to the following conjecture, which probably also occurred to other circle packers.

6.1. Conjecture. Let $T$ be an infinite triangulation with at most countably many ends. Then there exists a circle packing $P$ in $S^{2}$ whose nerve is $T$ and so that all the singularities of $P$ are either circles or single points. This packing is unique, up to Möbius transformations.

With the additional assumption that $T$ has bounded valence, the uniqueness part of this conjecture can be proved. The proof uses the techniques presented here, and the fact that in the bounded valence case, two packings with the same triangulation as nerve induce homeomorphisms between the boundaries of the corresponding singularities, provided these boundaries are simple closed curves. This fact follows from the analogous property for quasiconformal maps, and perhaps might also be true without the bounded valence restriction.

As mentioned in the introduction, the conjecture holds when $T$ has one end.

The observant reader may have noticed that our rigidity results for packings of convex sets other than circles do not deal with the case where infinity is (on the boundary of) a singularity of the packing. The reason for this is that we need homotheties to perturb the singularities, and the point at infinity is a fixed point for the homotheties. However, this difficulty does not rule out some kind of rigidity for packings having $\{\infty\}$ as a singularity. For example, one may ask:

6.2. Problem. Let $P$ and $Q$ be packings of smooth strictly convex bodies in the plane, both having a triangulation $T=T(V)$ as their nerve. Suppose that $\operatorname{carrier}(P)=\operatorname{carrier}(Q)=\mathbb{R}^{2} ; P_{v}$ is positively homothetic to $Q_{v}$ for each $v$; and $P_{v}=Q_{v}$ for $v=a, b, c$, where $[a, b, c]$ is some triangle of $T$. Does it follow that $Q=P$ ?

Added in proof. Conjecture 6.1 is true; the proof will appear in a joint work with Zheng-Xu He. 


\section{ACKNOWLEDGMENTS}

I am deeply thankful to my teachers Bill Thurston and Peter Doyle, and to Richard Schwartz, Burt Rodin, and Zheng-Xu He for stimulating discussions relating to packings.

\section{REFERENCES}

[An1] E. M. Andreev, On convex polyhedra in Lobacevskil spaces, Mat. Sb. (N.S.) 81 (1970), 445-478; English transl. in Math. USSR Sb. 10 (1970), 413-440.

[An2] - On convex polyhedra of finite volume in Lobacevskil space, Mat. Sb. (N.S.) 83 (1970), 256-260; English transl. in Math. USSR Sb. 12 (1970), 255-259.

[BFP] I. Bárány, Z. Füredi, and J. Pach, Discrete convex functions and proof of the six circle conjecture of Fejes Toth, Canad. J. Math. 36-3 (1984), 569-576.

[CR] I. Carter and B. Rodin, An inverse problem for circle packing and conformal mapping, preprint.

[He1] Zheng-Xu He, An estimate for hexagonal circle packings, J. Differential Geom. (to appear).

[He2] _ Solving Beltrami equations by circle packing, Trans. Amer. Math. Soc. (to appear).

[Ro1] B. Rodin, Schwartz's lemma for circle packings, Invent. Math. 89 (1987), 271-289.

[Ro2] _ Schwartz's lemma for circle packings II, J. Differential Geom. 30 (1989), 539-554.

[RS] B. Rodin and D. Sullivan, The convergence of circle packings to the Riemann mapping, J. Differential Geom. 26 (1987), 349-360.

[Sch1] O. Schramm, Packing two-dimensional bodies with prescribed combinatorics and applications to the construction of conformal and quasiconformal mappings, Ph.D. thesis, Princeton, 1990.

[Sch2] _ Uniqueness and existence of packings with specified combinatorics, Israel J. Math. (to appear).

[Ste] K. Stephenson, Circle packings in the approximation of conformal mappings, Bull. Amer. Math. Soc. 23 (1990), 407-415.

[Th1] W. P. Thurston, The geometry and topology of 3-manifolds, Princeton Univ. Lecture Notes, Princeton, NJ.

[Th2] _ The finite Riemann mapping theorem, invited talk at the International Symposium in Celebration of the Proof of the Bieberbach Conjecture, Purdue University, March 1985.

Department of Mathematics, Princeton University, Princeton, New Jersey, 08544

Current address: Mathematics Department, University of California at San Diego, La Jolla, California 92093

E-mail address: oschramm@math.ucsd.edu 